\title{
Thermokinetic lattice Boltzmann model of nonideal fluids
}

\author{
E. Reyhanian, ${ }^{1}$ B. Dorschner, ${ }^{1,2}$ and I. V. Karlin $\oplus^{1, *}$ \\ ${ }^{1}$ Department of Mechanical and Process Engineering, ETH Zurich, 8092 Zurich, Switzerland \\ ${ }^{2}$ California Institute of Technology, Pasadena, California 91125, USA
}

(Received 30 October 2019; accepted 4 August 2020; published 24 August 2020)

\begin{abstract}
We present a kinetic model for nonideal fluids, where the local thermodynamic pressure is imposed through appropriate rescaling of the particle's velocities, accounting for both long- and short-range effects and hence full thermodynamic consistency. The model features full Galilean invariance together with mass, momentum, and energy conservation and enables simulations ranging from subcritical to supercritical flows, which is illustrated on various benchmark flows such as anomalous shock waves or shock droplet interaction.
\end{abstract}

DOI: 10.1103/PhysRevE.102.020103

The lattice Boltzmann method (LBM) [1-4] is a modern approach to the simulation of complex flows. LBM is a recast of fluid mechanics into a kinetic theory for the populations of designer particles $f_{i}(\boldsymbol{x}, t)$, with simple rules of propagation on a space-filling lattice formed by discrete speeds $\mathcal{C}=\left\{\boldsymbol{c}_{i}\right.$, $i=1, \ldots, Q\}$, in discrete time $t$, and relaxation to a local equilibrium $f_{i}^{\text {eq }}(\boldsymbol{x}, t)$ at the nodes $\boldsymbol{x}$.

Classically, LBM features fluid dynamics as a fluctuation (subject to suitable nonlinearities) over a global thermodynamic reference state characterized by a lattice temperature $T_{\mathrm{L}}$ and a Galilean reference frame "at rest," $\boldsymbol{u}=\mathbf{0}$. While this viewpoint led to a successful LBM for incompressible flow, limitations are also apparent, in particular, for complex thermohydrodynamic processes for nonideal fluids. In order to address the entire spectrum of flows, from sub- to supersonic, involving sub- as well as supercritical processes, one needs a formulation of kinetic theory in a local rather than global thermodynamic reference frame [5].

In this Rapid Communication, we present a discretevelocity kinetic theory of nonideal fluids as an extension of recently proposed formalism [5]. Explicit realization of a local thermodynamic reference frame enables the simulation of demanding flow situations such as real-gas anomalous shock wave and droplet-shock interaction.

We follow [5] and define discrete velocities,

$$
\boldsymbol{v}_{i}=\sqrt{\frac{p}{\rho T_{\mathrm{L}}}} \boldsymbol{c}_{i}+\boldsymbol{u},
$$

where $p(\boldsymbol{x}, t)$ is the local thermodynamic pressure, $\rho(\boldsymbol{x}, t)$ is the local density, and $T_{\mathrm{L}}$ is a lattice reference temperature, a constant known for any set of speeds $\mathcal{C}$, and $\boldsymbol{u}(\boldsymbol{x}, t)$ is the local flow velocity. While (1) applies to a generic equation of state (EOS) $p$, below we adopt that of the van der Waals, $p=\rho R T /(1-b \rho)-a \rho^{2}$, with critical values $\rho_{\mathrm{cr}}=1 / 3 b$, $T_{\mathrm{cr}}=8 a / 27 R b, p_{\mathrm{cr}}=a /\left(27 b^{2}\right)$; we set $a=2 / 49, b=2 / 21$, and $R=1$ in the simulations.

\footnotetext{
*Corresponding author: ikarlin@ethz.ch
}

Local fields in Eq. (1) are evaluated using two sets of populations, $f$ and $g$; the former maintains the density and momentum while the latter corresponds to energy conservation:

$$
\rho=\sum_{i=1}^{Q} f_{i}, \quad \rho \boldsymbol{u}=\sum_{i=1}^{Q} f_{i} \boldsymbol{v}_{i}, \quad 2 \rho E=\sum_{i=1}^{Q} g_{i},
$$

where $E$ is the energy per unit mass, $E=u^{2} / 2+e$, where $e=e(s, v)$ is the local internal energy per unit of mass, $s$ is the entropy, $v=1 / \rho$ the specific volume, and temperature is defined by the thermodynamic relation, $T=(\partial e / \partial s)_{v}$.

With the particles' velocities rescaled by the local thermodynamic pressure, Eq. (1) and the local conservation of the mass, momentum, and energy, Eq. (2), we are set to formulate a kinetic model which, neglecting the interface energy in the two-phase coexistence region in the first instance, shall be essentially local in the sense of the conventional LBM. Same as in LBM, the proposed scheme is split into two parts: streaming and collision. Semi-Lagrangian advection [5] is adopted in the streaming step:

$$
\begin{aligned}
& f_{i}(\boldsymbol{x}, t)=f_{i}\left(\boldsymbol{x}-\boldsymbol{v}_{i} \delta t, t-\delta t\right), \\
& g_{i}(\boldsymbol{x}, t)=g_{i}\left(\boldsymbol{x}-\boldsymbol{v}_{i} \delta t, t-\delta t\right) .
\end{aligned}
$$

The collision step is then performed employing the BhatnagarGross-Krook (BGK) model,

$$
\begin{gathered}
f_{i}^{*}(\boldsymbol{x}, t)=f_{i}(\boldsymbol{x}, t)+\omega\left[f_{i}^{\mathrm{eq}}-f_{i}(\boldsymbol{x}, t)\right], \\
g_{i}^{*}(\boldsymbol{x}, t)=g_{i}(\boldsymbol{x}, t)+\omega\left[g_{i}^{\mathrm{eq}}-g_{i}(\boldsymbol{x}, t)\right]+G_{i} \delta t .
\end{gathered}
$$

The local equilibrium populations in the comoving reference frame reduce to

$$
\begin{gathered}
f_{i}^{\mathrm{eq}}=\rho W_{i}, \\
g_{i}^{\mathrm{eq}}=\rho W_{i}\left[2 e-D(p / \rho)+v_{i}^{2}\right],
\end{gathered}
$$

where $W_{i}$ are conventional LBM lattice weights known for any set of discrete speeds $\mathcal{C}$. The term $G_{i} \delta t$ is introduced in Eq. (6) 
in order to correct the heat flux,

$$
G_{i}=M_{0} W_{i}\left(1+\frac{\rho\left(\boldsymbol{u} \cdot \boldsymbol{c}_{i}\right)^{2}}{2 p T_{\mathrm{L}}}-\frac{\rho v_{i}^{2}}{2 p}+\frac{D}{2}\right),
$$

where $M_{0}=2 \nabla \cdot(-\mu \nabla h+k \nabla T), \mu$ is the shear viscosity, $h=e+p / \rho$ is the enthalpy, and $k$ is the thermal conductivity which can be set independently.

The kinetic system (3)-(8) recovers the equations for density, flow velocity, and temperature in the hydrodynamic limit as follows:

$$
\begin{gathered}
D_{t} \rho=-\rho \nabla \cdot \boldsymbol{u}, \\
\rho D_{t} \boldsymbol{u}=-\nabla p-\nabla \cdot \boldsymbol{\tau}, \\
\rho C_{v} D_{t} T=-\boldsymbol{\tau}: \nabla \boldsymbol{u}-T\left(\frac{\partial p}{\partial T}\right)_{v} \nabla \cdot \boldsymbol{u}-\nabla \cdot \boldsymbol{q},
\end{gathered}
$$

where $D_{t}=\partial_{t}+\boldsymbol{u} \cdot \nabla$ is the material derivative, $C_{v}=$ $(\partial e / \partial T)_{v}$ is the specific heat at constant volume, $\boldsymbol{q}=-k \nabla T$ is the heat flux, and the nonequilibrium stress tensor reads

$$
\boldsymbol{\tau}=-\mu\left(\nabla \boldsymbol{u}+\nabla \boldsymbol{u}^{\dagger}-\frac{2}{D}(\nabla \cdot \boldsymbol{u}) \boldsymbol{I}\right)-\eta(\nabla \cdot \boldsymbol{u}) \boldsymbol{I}
$$

The shear and bulk viscosity are

$$
\begin{gathered}
\mu=\left(\frac{1}{\omega}-\frac{1}{2}\right) p \delta t \\
\eta=\left(\frac{1}{\omega}-\frac{1}{2}\right)\left(\frac{D+2}{D}-\frac{\rho \varsigma^{2}}{p}\right) p \delta t
\end{gathered}
$$

respectively, and $\varsigma=\sqrt{(\partial p / \partial \rho)_{s}}$ is the speed of sound. Note that the bulk viscosity vanishes in the limit of ideal monatomic gas, $p \rightarrow \rho R T, \varsigma^{2} \rightarrow(D+2) R T / D$, as expected.

While the derivation of the hydrodynamic limit follows the standard analysis (see Supplemental Material for details [6]), a comment on the difference of our proposal from a more microscopic Vlasov-Enskog kinetic equation is in order. The latter employs a short-range, excluded volume collision (Enskog) along with a mean-field force representing a long-range attraction (Vlasov) (see, e.g., [7]). One prominent feature of the Vlasov-Enskog model is its nonlocality: the momentum and energy are conserved globally but not locally. This is in contrast not only to the Boltzmann equation but also to the macroscopic fluid dynamics equations, which are local in the sense of thermodynamic pressure and energy fields [see Eqs. (11) and (12)]. The latter can be derived from the VlasovEnskog equation once the nonlocality is moderated through a corresponding gradient expansion; with this, the combined action of both Vlasov and Enskog terms eventually leads to a van der Waals-type equation of state. The conventional scenario to derive a reduced model from the Vlasov-Enskog kinetic equation is to use (a) the Boltzmann or BGK collision as the leading locally conserving term and define the particles velocities through the conventional (Gauss-Hermite) sampling of the local Maxwellian, and (b) to evaluate the remaining (i.e., essentially nonlocal) parts by the same quadrature. The latter step brings in all of the nonideal fluid thermodynamics as a nonlocal forcing term added to the leading local collision (see, e.g., [8]).

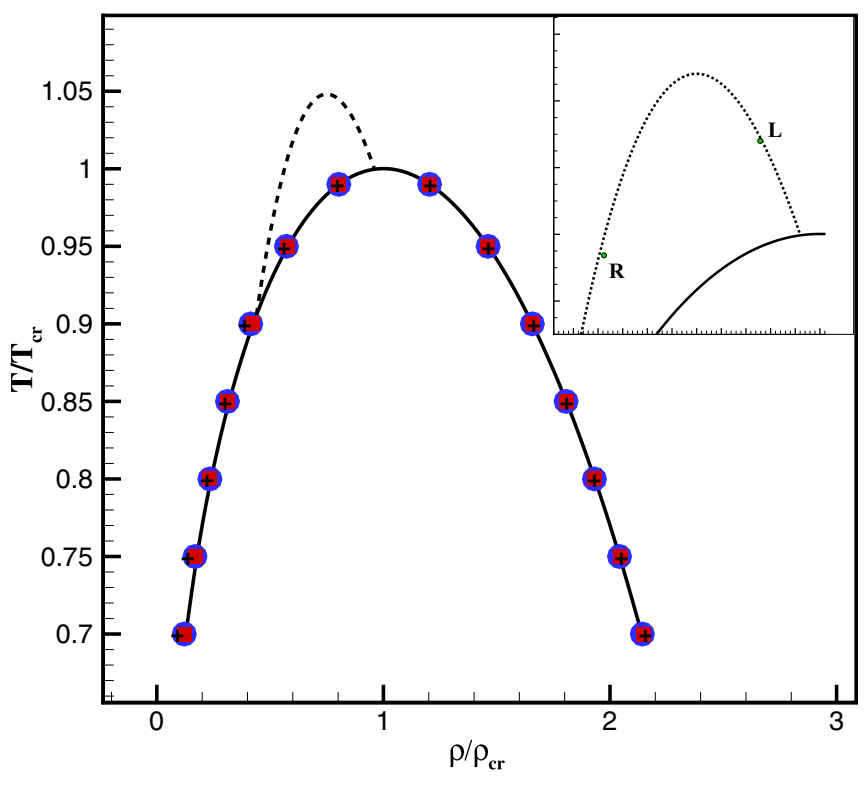

FIG. 1. Coexistence curve of a van der Waals fluid. Solid line: Theory (Maxwell's equal area rule). Symbol: Simulation. Circle: $\delta=1 / 3$. Square: $\delta=2 / 3$. Cross: $\delta=1 / 3$ advected with the speed $U=5 U_{\mathrm{ch}}$, characteristic velocity $U_{\mathrm{ch}}=\sqrt{p_{\mathrm{cr}} / \rho_{\mathrm{cr}}}$. Dashed line: Zero line of fundamental derivative, $\Gamma=0$, for $\delta=0.0125$ (theory). Inset shows the left and right conditions in the simulation of the shock-tube problem in Fig. 3.

Here we take a different, bottom-up route to extend the target hydrodynamic equations to a local (in the sense of the conventional lattice BGK) kinetic model. This is achieved by two considerations: First, we employ the scaling of the particles' velocities by the local thermodynamic pressure, Eq. (1). This step endows the manifestly Galilean invariant momentum equation (11) with the target thermodynamic pressure of nonideal fluid. We term our model "thermokinetic" in order to stress that particles' velocities are established from the target local thermodynamics. Second, we maintain the total energy conservation, again in a local fashion, by the second populations. Certainly, the locality approach has its limitations. First, we cannot avoid nonlocality entirely but it only appears as a forcing term, which implements the surface tension in the two-phase region (see below). Second, the heat flux, resulting from the local energy conservation [Eq. (6) with the last term dropped] would be proportional to the gradient of the enthalpy, $\boldsymbol{q}=-\mu \nabla h$, rather than temperature. The heat flux is corrected by introducing a counterterm $G_{i} \delta t$ in Eq. (6).

We now proceed with the validation of the thermodynamic features of the proposed kinetic model. The standard $D 2 Q 9$ lattice $\mathcal{C}=\mathcal{C}_{1} \otimes \mathcal{C}_{1}$, where $\mathcal{C}_{1}=\{-1,0,1\}$ was used in all simulations. For the van der Waals fluid, the internal energy is given by $e=C_{v} T-a \rho$ and the specific heat at constant volume is $C_{v}=R / \delta$, where $0<\delta \leqslant 2 / 3$ [9-11]. Figure 1 demonstrates the independence of saturated liquid and vapor densities on the choice of $\delta$, also in a moving reference frame; results are in excellent agreement with Maxwell's equal area rule.

Furthermore, speed of sound was measured by introducing a pressure disturbance $\delta p=10^{-3}$ in the liquid-gas phases 


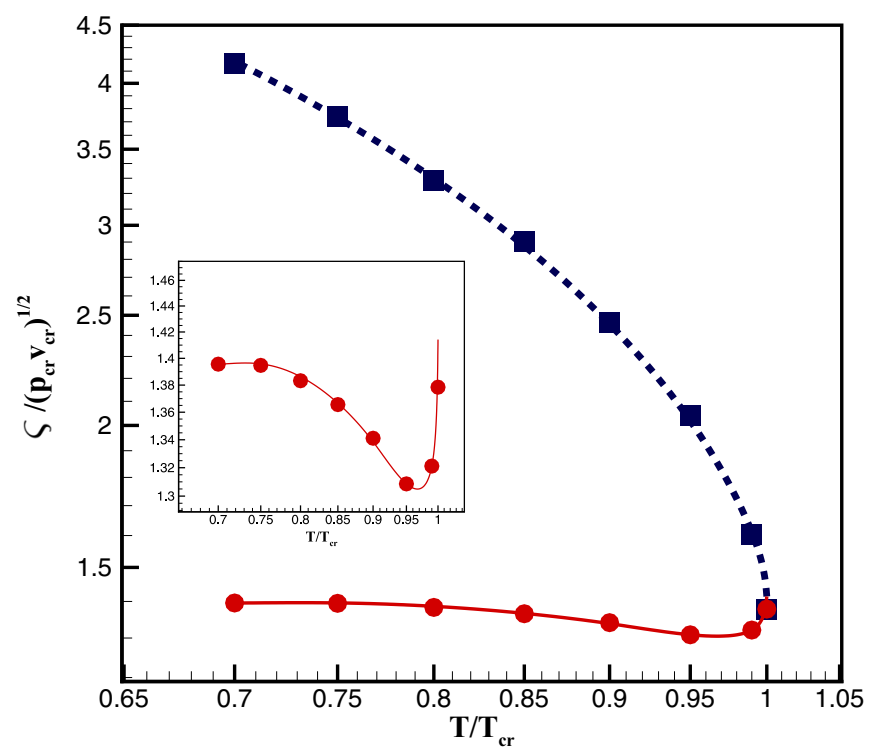

FIG. 2. Reduced speed of sound in the van der Waals fluid with $\delta=1 / 3$ as a function of the reduced temperature. Solid line: Saturated vapor (theory). Dashed line: Saturated liquid (theory). Symbol: Simulation. Inset shows details of the speed of sound in the vapor near critical point.

assumed at the saturation, and tracking the resulting shock front. Simulation results compare well with the theoretical prediction $\varsigma^{2}=\left(C_{p} / C_{v}\right)(\partial p / \partial \rho)_{T}$ in Fig. 2. Note that the simulation predicts the speed of sound correctly at critical point $\zeta_{\mathrm{cr}}=\sqrt{(6 \delta)\left(p_{\mathrm{cr}} v_{\mathrm{cr}}\right)}$. The latter result is nontrivial because finiteness of the speed of sound at critical point is maintained by the simultaneous divergence of $C_{p}$ and vanishing of the derivative $(\partial p / \partial \rho)_{T}$. The inset in Fig. 2 demonstrates a nonmonotonic behavior of the speed of sound in the vapor phase for sufficiently large $C_{v}(\delta=1 / 3)$ : a decrease with the increase of the temperature up to $T / T_{\mathrm{cr}}=0.95$ followed by a sharp increase, matching the liquid line at the critical point.

Finally, we consider the anomalous shock-wave problem [10,12-14]. For ideal gas, only compression shocks and rarefaction waves are admissible. However, in a nonideal gas (Bethe-Zel'dovich-Thompson fluid), a region near the vapor saturation line may exist, where a rarefaction shock traveling in the direction of the increase of pressure can be observed. This anomalous behavior is characterized by the so-called fundamental derivative, $\Gamma=\left(v^{3} / 2 \varsigma^{2}\right)\left(\partial^{2} p / \partial v^{2}\right)_{s}$. An anomalous shock occurs when $\Gamma<0$ and can be modeled with the van der Waals fluid at large specific heat values (see inset in Fig. 1). Figure 3 shows a snapshot of density and pressure profiles in the shock tube simulation when the left and right ends of the domain are in the $\Gamma<0$ domain. The anomalous rarefaction shock is clearly visible, traveling towards the high pressure end (left) together with a compression wave propagating into the low pressure region (right). The comparison between the present scheme and Ref. [13] shows good agreement.

We now finalize the model development by including the effect of the liquid-vapor interface. This is done in two steps. First, we modify the $f$-populations kinetics in a conventional fashion by introducing a forcing term $S_{i}$ after the collision

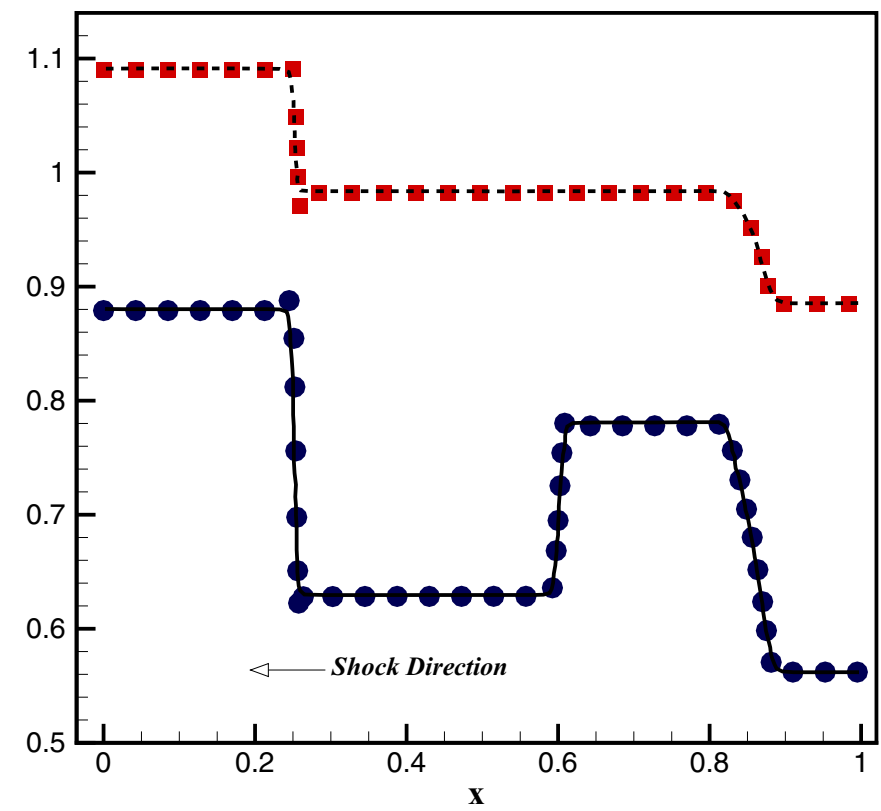

FIG. 3. Simulation of an anomalous shock in the van der Waals fluid, $\delta=0.0125$. Initial conditions: $\left(p_{\mathrm{L}}, \rho_{\mathrm{L}}\right)=(1.09,0.879)$, $\left(p_{\mathrm{R}}, \rho_{\mathrm{R}}\right)=(0.885,0.562)$ were applied to the left and right parts of the tube. The snapshot is taken at time $t^{*}=(t / L) \sqrt{p_{\mathrm{cr}} / \rho_{\mathrm{cr}}}=0.45$, where $L$ is the length of the domain. Line: Density [13]. Dashed: Pressure [13]. Symbol: Present.

step (5),

$$
f_{i}^{*}(\boldsymbol{x}, t)=f_{i}(\boldsymbol{x}, t)+\omega\left[\rho W_{i}-f_{i}(\boldsymbol{x}, t)\right]+S_{i},
$$

where

$$
S_{i}=\mathcal{G}_{\boldsymbol{u}+\delta \boldsymbol{u}}^{\boldsymbol{u}}\left[\rho W_{i}\right]-\rho W_{i} .
$$

Here $\mathcal{G}$ is the transformation matrix (see [5] and Supplemental Material [6]) and $\delta \boldsymbol{u}=(\mathbf{F} / \rho) \delta t$ is the change of the local flow velocity due to the force $\mathbf{F}=\nabla \cdot \boldsymbol{K}$, where

$$
\boldsymbol{K}=-\kappa\left(\rho \nabla \cdot \nabla \rho+\frac{1}{2}|\nabla \rho|^{2}\right) \boldsymbol{I}+\kappa(\nabla \rho)(\nabla \rho)
$$

is the Korteweg stress and $\kappa$ is the surface tension coefficient [15]. One can readily recognize that the forcing (17) is the exact difference method $[16,17]$ adapted to the comoving reference frame [5]. Thus, the actual fluid velocity is redefined as $\hat{\boldsymbol{u}}=\boldsymbol{u}+\delta \boldsymbol{u} / 2$. With this, the momentum equation (11) becomes

$$
\rho \hat{D}_{t} \hat{\boldsymbol{u}}=-\nabla p-\nabla \cdot \hat{\boldsymbol{\tau}}-\nabla \cdot \boldsymbol{K},
$$

where $\hat{\boldsymbol{\tau}}$ is the stress tensor (13) based on the actual flow velocity $\hat{\boldsymbol{u}}$, and $\hat{D}_{t}=\partial_{t}+\hat{\boldsymbol{u}} \cdot \nabla$.

Second, the $g$-populations kinetics are modified accordingly. To that end, the local energy $\rho E$ is redefined with respect to the actual flow velocity, $\rho \hat{E}=\rho e+\rho \hat{u}^{2} / 2$. Consequently, the local equilibrium (8) is modified to take into account the actual flow velocity,

$$
g_{i}^{\mathrm{eq}}=W_{i}\left[2 \rho \hat{E}+\boldsymbol{M} \cdot\left(\boldsymbol{v}_{i}-\boldsymbol{u}\right)+\boldsymbol{N}:\left(\boldsymbol{v}_{i} \boldsymbol{v}_{i}-\frac{p}{\rho} \boldsymbol{I}-\boldsymbol{u} \boldsymbol{u}\right)\right],
$$




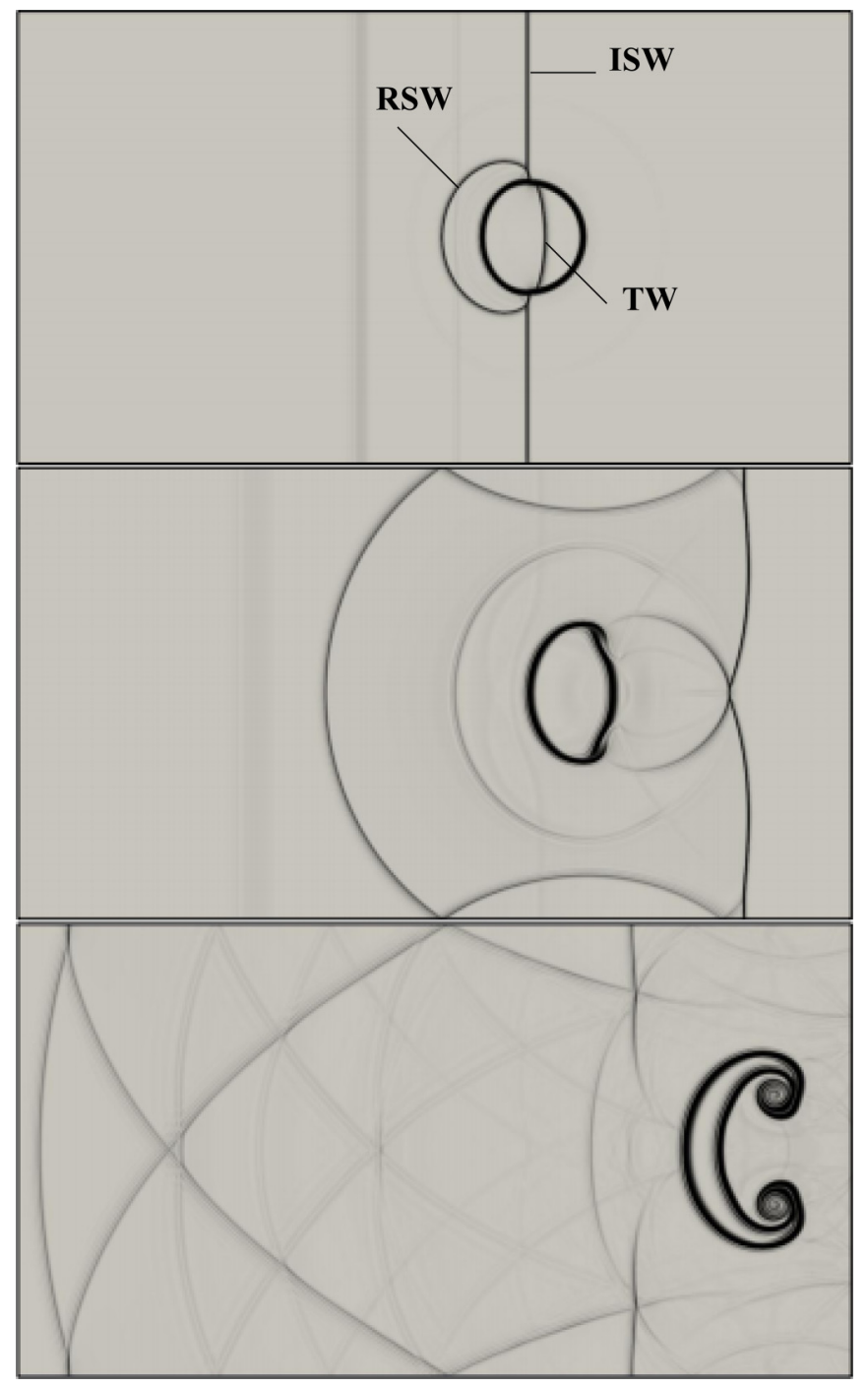

FIG. 4. Schlieren images of the interaction of a shock wave at $\mathrm{Ma}=1.47$ and a droplet at different times using the present scheme. From top to bottom: $t^{*}=0.298, t^{*}=0.879, t^{*}=2.413$.

where

$$
\begin{gathered}
\boldsymbol{M}=\frac{\delta t}{(p / \rho)}\left[\boldsymbol{F}\left(\hat{E}+\frac{p}{\rho}\right)-\boldsymbol{G} \cdot \boldsymbol{u}\right], \\
\boldsymbol{N}=\rho \boldsymbol{I}+\frac{\delta t}{(2 p / \rho)} \boldsymbol{G}, \\
\boldsymbol{G}=\hat{\boldsymbol{u}} \boldsymbol{F}+\boldsymbol{F} \hat{\boldsymbol{u}}+\delta t \boldsymbol{F} \boldsymbol{F} \frac{\hat{E}}{2 p} .
\end{gathered}
$$

Note that in the absence of the force, the equilibrium (20) simplifies to Eq. (8). Finally, the correction term (9) is also modified in the presence of the Korteweg stress, $M_{0}=2 \nabla$. $(-\mu \nabla h+k \nabla T)+2 \hat{\boldsymbol{u}} \cdot \nabla \cdot \boldsymbol{K}$.

Apart from the local energy $\rho \hat{E}$, the total energy of a twophase system $\rho \hat{\mathcal{E}}$ also includes the energy of the interface,

$$
\rho \hat{\mathcal{E}}=\rho \hat{E}+\frac{\kappa}{2}|\nabla \rho|^{2} .
$$

With the above changes to the $g$-populations kinetics, the equation for the total energy is recovered in a standard form

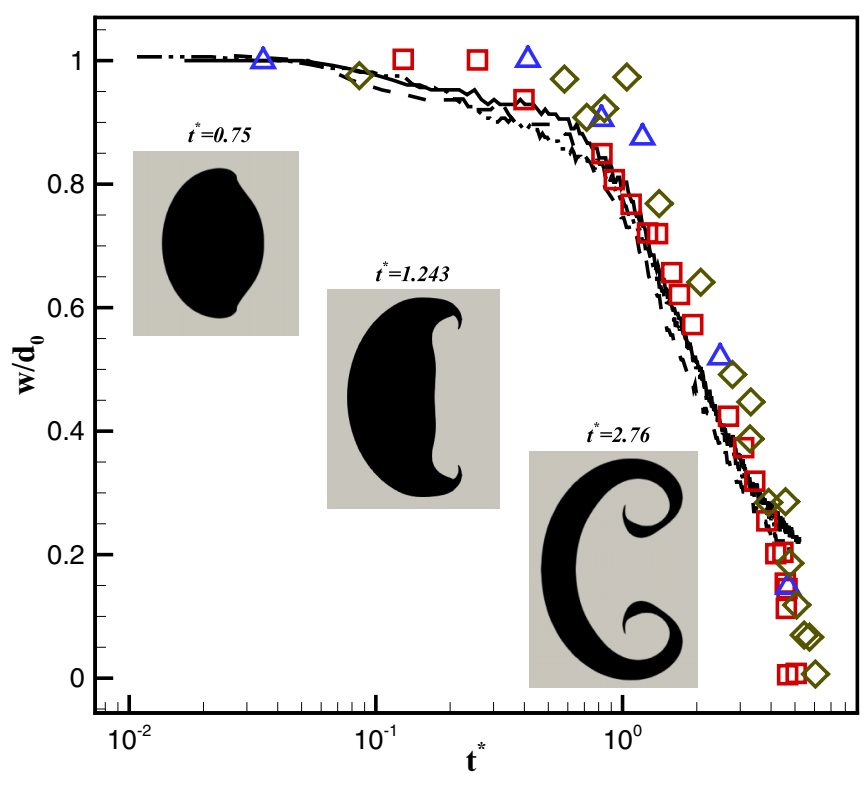

FIG. 5. Two-dimensional droplet center-line width evolution. Lines: Present scheme. Solid line: $M_{s}=1.18$. Long dashed: $M_{s}=$ 1.33. Dashed: $M_{s}=1.47$. Symbol: Experiment [22]. Square: $M_{s}=$ 1.18. Delta: $M_{s}=1.30$. Diamond: $M_{s}=1.47$. Inset: Cross section of the droplet at three different times.

for a two-phase system (e.g., [18]; see Supplemental Material for details [6]),

$$
\begin{gathered}
\partial_{t}(\rho \hat{\mathcal{E}})+\nabla \cdot(\rho \hat{\mathcal{E}} \hat{\boldsymbol{u}}+p \hat{\boldsymbol{u}}+\hat{\boldsymbol{\tau}} \cdot \hat{\boldsymbol{u}}+\boldsymbol{q}) \\
+\nabla \cdot(\boldsymbol{K} \cdot \hat{\boldsymbol{u}}+\kappa \rho \nabla \cdot \hat{\boldsymbol{u}} \nabla \rho)=0 .
\end{gathered}
$$

However, the density (10) and the temperature (12) equations remain intact provided $\boldsymbol{u}$ is replaced by the actual flow velocity $\hat{\boldsymbol{u}}$. The interface has no effect on the thermodynamic temperature equation, as is expected [18].

We conclude this Rapid Communication with a simulation of the interaction of a water column with a planar shock wave. To this end, a planar shock wave was generated initially separating the postshock part and the saturated vapor. A twodimensional droplet representing a cross section of the liquid column is placed downstream from the shock, initially at equilibrium with the vapor at the temperature $T / T_{\mathrm{cr}}=0.9$. In order to compare with the experiment $[19,20]$, we use the reduced time $t^{*}=t\left(u_{g} / d_{0}\right) \sqrt{\rho_{\mathrm{g}} / \rho_{\mathrm{l}}}\left(\varsigma_{l} / \varsigma_{g}\right)$ where $\varsigma_{l, g}$ is the speed of sound in the liquid and the postshock gas, $\rho_{l, g}$ are corresponding densities, $d_{0}$ is the initial diameter of the liquid column, and $u_{g}$ is the flow speed upstream.

The evolution of the water-column cross section is shown in Fig. 4 by Schlieren images. After the incident shock wave (ISW) reaches the upstream interface of the droplet, one can see that a left-propagating reflected shock wave (RSW) as well as the transmitted wave (TW) are generated. The TW quickly travels the width of the droplet since the speed of sound is significantly higher in the liquid phase than in the vapor. These are typical waves generated in the early stages upon the impingement of a shock wave on a droplet as reported in experiments and other numerical simulations [19,21]. At later stages, the droplet starts flattening in the flow direction and expanding in the radial direction. Furthermore, two vortices 
are formed near the equator, which are the result of the shear forces and the flow separation behind the droplet. Figure 5 represents a more quantitative assessment of the simulation where the width of the droplet was measured with respect to its center line and compared to the experimental results from [22]. The results are in good agreement with the experiment showing that the deformation of the droplet was accurately captured by the proposed scheme.

In conclusion, the main novelty of our approach is to introduce local thermodynamics by a velocity rescaling whereas the rest follow automatically provided the local conservation laws are correctly taken into account by model collision. The sampling of particles' velocities based on the local thermodynamics is markedly different from a conventional GaussHermite sampling at the core of LBM. In our notation, LBM velocities correspond to choosing the ideal gas pressure at a constant lattice temperature $p_{\mathrm{L}}=\rho T_{\mathrm{L}}$ in Eq. (1) whereas we proposed the target thermodynamic pressure therein instead. The restriction of the flow velocity in LBM to small values limits its application in high-velocity flows, leaving the compressible-multiphase flow regime an uncharted field in the context of LBM. The Galilean-invariant nature of this method removes this barrier, on account of error-free collision. Realgas EOS is naturally introduced through the discrete particle velocities, providing full thermodynamic consistency. Finally, we note that the semi-Lagrangian propagation adopted in the present numerical realization can alternatively be replaced by other available off-lattice propagation schemes such as finite volumes or finite differences. The above benchmarks demonstrate that the proposed approach can be useful to study dynamical regimes of nonideal fluids in the supercritical and supersonic flow regimes which are not readily accessible by standard LBM. Exploring these flow regimes shall be the focus of our future studies.

The authors thank F. Bösch for the helpful discussions. This work was supported by the European Research Council (ERC) Advanced Grant No. 834763-PonD and the Swiss National Science Foundation (SNSF) Grants No. 200021172640 (E.R.) and No. P2EZP2_178436 (B.D.). Computational resources at the Swiss National Super Computing Center (CSCS) were provided under Grant No. s897.
[1] U. Frisch, B. Hasslacher, and Y. Pomeau, Phys. Rev. Lett. 56, 1505 (1986).

[2] G. R. McNamara and G. Zanetti, Phys. Rev. Lett. 61, 2332 (1988).

[3] F. J. Higuera, S. Succi, and R. Benzi, Europhys. Lett. 9, 345 (1989).

[4] Y.-H. Qian, D. d'Humières, and P. Lallemand, Europhys. Lett. 17, 479 (1992).

[5] B. Dorschner, F. Bösch, and I. V. Karlin, Phys. Rev. Lett. 121, 130602 (2018).

[6] See Supplemental Material at http://link.aps.org/supplemental/ 10.1103/PhysRevE.102.020103 for derivation of the hydrodynamic limit of the model, thermodynamical relations, and more simulations.

[7] J. Karkheck and G. Stell, J. Chem. Phys. 75, 1475 (1981).

[8] X. He and G. D. Doolen, J. Stat. Phys. 107, 309 (2002).

[9] J. Liu, in Frontiers in Computational Fluid-Structure Interaction and Flow Simulation (Springer, New York, 2018), pp. 377-398.

[10] N. Zhao, A. Mentrelli, T. Ruggeri, and M. Sugiyama, Phys. Fluids 23, 086101 (2011).
[11] P. Colonna and A. Guardone, Phys. Fluids 18, 056101 (2006).

[12] J. W. Bates and D. C. Montgomery, Phys. Fluids 11, 462 (1999).

[13] A. Guardone and L. Vigevano, J. Comput. Phys. 175, 50 (2002).

[14] C. Zamfirescu, A. Guardone, and P. Colonna, J. Fluid Mech. 599, 363 (2008).

[15] A. Mazloomi M, S. S. Chikatamarla, and I. V. Karlin, Phys. Rev. Lett. 114, 174502 (2015).

[16] A. L. Kupershtokh, Vestn. Novosib. Gos. Univ., Ser.: Mat., Mekh., Inform 4, 75 (2004).

[17] A. L. Kupershtokh, D. A. Medvedev, and D. I. Karpov, Comput. Math. Appl. 58, 965 (2009).

[18] F. Magaletti, L. Marino, and C. M. Casciola, Phys. Rev. Lett. 114, 064501 (2015).

[19] G. Xiang and B. Wang, J. Fluid Mech. 825, 825 (2017).

[20] D. Igra and K. Takayama, J. Fluids Eng. 125, 325 (2003).

[21] S. Sembian, M. Liverts, N. Tillmark, and N. Apazidis, Phys. Fluids 28, 056102 (2016).

[22] D. Igra and K. Takayama, A study of shock wave loading on a cylindrical water column, Tech. Rep., Institute of Fluid Science, Tohoku University (2001), Vol. 13, pp. 19-36. 